\title{
A Case Study on Implementing Future Human-Machine Interfaces
}

\author{
Ljubo Mercep, Gernot Spiegelberg and Alois Knoll
}

\begin{abstract}
In the scope of the Diesel Reloaded project, we conducted a study on future automotive human-machine interfaces (HMI) with an overview of their relationship to driver assistance systems (DAS). Furthermore, we implemented a series of HMI and DAS concepts in our prototype vehicle and in a modified driving simulator. Emphasis was placed on the following goals: Pushing the complexity away from the driver and inside the intelligent vehicle, developing unified and extendable descriptions of interaction context, defining transitional steps to the long-term goal of user interfaces which augment the driver, leveraging cross-domain technology transfer and addressing relevant societal trends. In this work, we provide a top-level overview of our results and conclusions we drew based upon our two-year research and prototype construction and deployment in the area of human-machine interfaces and driver assistance.
\end{abstract}

\section{INTRODUCTION}

The project Diesel Reloaded is a cooperation with a series of industry and research partners hosted on the Institute of Advanced Study and International Graduate School of Science and Engineering of the Technische Universität München. It addresses the research areas of system architecture, energy management, human-machine interface and driver assistance. Emphasis is placed on an interdisciplinary approach to all the research questions, which are analyzed from both engineering and scientific point of view. During the first two years, a fully-functional vehicle prototype, the Innotruck, shown in figure 1, has been constructed and tested on road [1]. It features all the key elements of the so-called transitional future interfaces, as the first step in a long term transformation of the driver's workspace. In parallel, a driving simulator has been modified in order to implement some elements of the next evolutionary step and rapidly collect and process test data in a virtual environment.

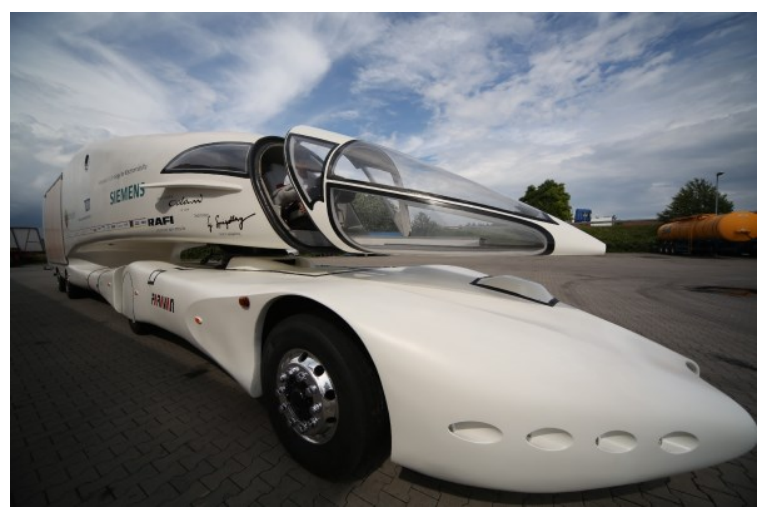

Figure 1. The project demonstrator for the first evolutionary step in interface development - the Innotruck.
In a sense, this research extends and builds upon the results of the Adaptive Integrated Driver-Vehicle Interface project from the Sixth Framework Programme. AIDE placed emphasis on the functional growth in the driving environment, taking driver assistance systems and information systems into account [2]. Driver distraction and physical integration were thoroughly analyzed. The information flow to the driver was to be centralized and adapted to the current situation. A real-time monitoring of driver-vehicle-environment state was therefore necessary. Our work, on the other side, is focused on the effects of the projected technology shift in the area of interfaces for continuous vehicle control, moving towards less physically intensive and more integrated input methods. All the topics analyzed during AIDE are reopened in this new context. Some aspects of this work are directly transferrable to robotics and other non-automotive areas of interest.

This paper is organized as follows. In chapter II we give an overview of the current state of HMI design. In chapter III we address some of the trends relevant to our approach. In chapter IV we give an overview of importance of context in human communication. Chapter $\mathrm{V}$ gives an overview of the important aspects of system architecture. In chapter VI we provide guidelines for handling the future HMI solutions which are then partially implemented in the prototype in chapter VII and in the simulator in chapter VIII. Finally, we conclude and present future work in chapter IX

\section{CONTEMPORARY HUMAN-MACHINE INTERFACES}

Current automotive interfaces are built upon a so-called vertical approach, where each physical interface component directly controls a certain vehicle part. Brakes and throttle are, for example, both controlling the longitudinal vehicle dynamics through separated control of two different subsystems. An example is given in figure 2 .

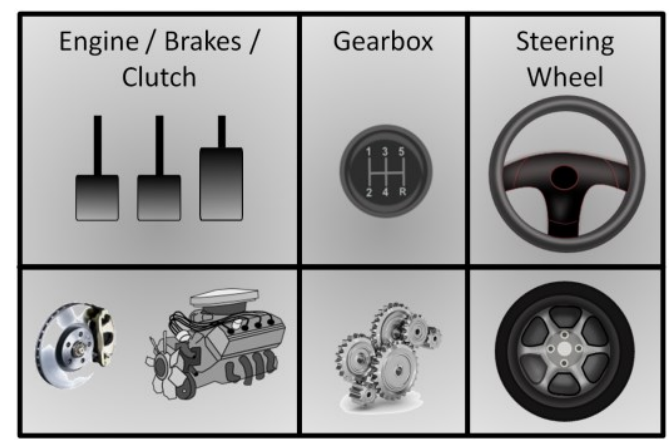

Figure 2. Example of a vertical interface design.

The issues we see with this approach are twofold. Firstly, the vehicle control system is strongly tied to the vehicle type. A looser coupling, made possible by the addition of a middle 
interpretation layer between the driver and the actual drivetrain, would allow for a control system transferrable between various vehicle types. It would additionally allow a unified interpretation level, instead of processing separated interface inputs. Of course, a drive-by-wire system is necessary in order to implement this kind of vehicle control. The second issue is the physical force and overall physical engagement necessary to operate the vehicle. It can be a problem in elder population or with people who are physically impaired.

Future approach, on the other hand, tries to maximize the information transferred between the human and the machine over minimal physical effort. To achieve this goal, the interpretation layer has to process as much situational context as possible, in order to appropriately interact with the user. The context also contains user profiles, personal preferences and their level of acquaintance with the technical system. The resulting horizontal design does not only affect driver's input, but also includes the feedback channels from the vehicle to the driver. Virtualized dashboards are an example of such integrated feedback solutions, as opposed to various physical systems with their own status indicators.

\section{RELEVANT MEGATRENDS}

In this chapter we present a series of global trends currently which are addressed by our approach. Leveraging such trends can provide public awareness and funds during the transitional interface development.

\section{A. Demographic Shift}

Aging population is having more difficulties operating a conventional physically-intensive vehicle. Seniors should retain their personal mobility in situations where fully autonomous driving is not possible. Special consideration has to be made when building the assistance systems which adapt to the user's state, allowing for lower tolerances and discrepancies between the optimal and real vehicle control. On the other side, the younger generation wants more functionality from their vehicle and wants to experience the full spectrum of HMI applications.

\section{B. Personal Mobility as a Service}

Combined mobility solutions, like using railway together with car or bike-sharing, can completely change the automotive landscape of the future. In this scenario, personal mobility is provided without owning the vehicle, via a shared fleet of personal automobiles. Personal parameterization of the human-machine interface and overall vehicle look and feel can be transferred upon entering the vehicle. This can be taken one step further, to semi-autonomous or autonomous operation of a pool of vehicles. Ordering of a vehicle to a specific location could easily be done via smartphone. The same personal profile could now be used for entertainment and socializing purposes.

\section{Urban Growth}

The ever-increasing population in urban areas will result with new personal mobility paradigms. Vehicles might include semi-autonomous operation for parking maneuvers, especially when coupled with charging process in large parking houses for electrical vehicles. Learning and supportive applications for maneuver assistance can increase safety on tightly packed streets and help the driver plan his route in the urban jungle.

\section{HUMAN INTERFACE SPECIFICS}

When considering the modalities for human data input and machine feedback, several aspects have to be considered. The channels from the human to the machine have for now been mostly discrete and very low-bandwidth. This not only lies on the semantics of human-machine interfaces, but also on the technology behind the implemented physical interfaces. When two humans communicate through any low-bandwidth channel, the amount of actual exchanged information can be huge due to the context and interpretation of indirect channels such as body talk. Future interfaces will be able to extract (based on advances in signal processing) and understand (based on advances in data mining and processing techniques) such additional data. In this sense, the acquisition and understanding of information context coming from the HMI will be a large factor in providing new functionality and interaction modes. The second challenge lies by the efficient modeling of the driver-vehicle-environment construct and efficient inference on such model, which can answer situation-relevant questions.

\section{System ARCHITECTURE CHANGES}

This chapter briefly addresses the architectural changes related to the HMI and DAS and how they translate to the Innotruck and its system architecture. A more general overview of future vehicle architectures is given in [3]. The middle layer between the actual physical HMI and the command execution on the system side processes a growing amount of contextual information. This is necessary in order to correctly interpret user's wish and perform all the safety checks before executing a command which could endanger the user. The control over the feedback channels towards the driver will require the same contextual-based processing to provide the right amount of relevant information. These requirements are now fitted inside the Innotruck's system architecture, based on Prof. Spiegelberg's 5-Module approach. This approach is chosen as a higher-level principle of organizing the system components inside larger modules. Design of modules follows, among other ideas, the principle of minimizing the inter-module data transfer overhead. When implementing the modules in hardware, the amount of data being exchanged on a common bus would therefore be close to minimal. Figure 3 provides an overview of the main modules: HMI, DAS, Drivetrain and Comfort Systems. The fifth one, painted in red, provides the infrastructure necessary for the 4 other modules to function. Further submodules like the virtual co-driver and the arbitration unit are shown only as an example of current functionality of the driver assistance. The 5-Module approach itself is not a contribution of this work and is not explained in further detail. 


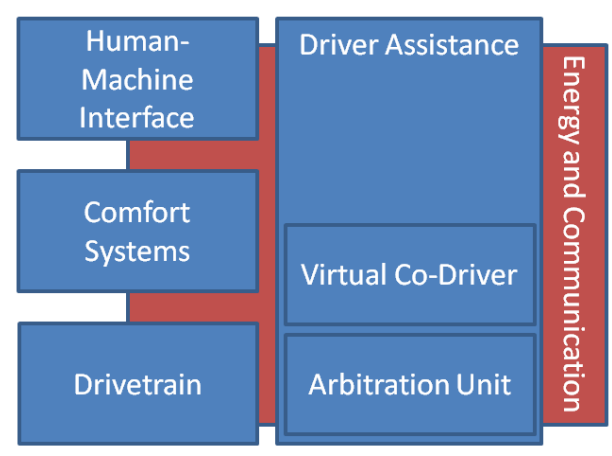

Figure 3. Overview of the 5-Module approach

The introduction of contextual data, which is necessary for the middle layer, results in a slight change of roles. There will be no "direct" communication between the elements of the HMI with other modules. Every HMI interaction will have to be refined with context data and reacted to accordingly. This results in an evolution of the driver assistance module to a more general driver extension or driver augmentation module, absorbing the HMI as one of the data sources and sinks. The new proposed module configuration, as an evolution of the 5-module approach, is shown in figure 4.

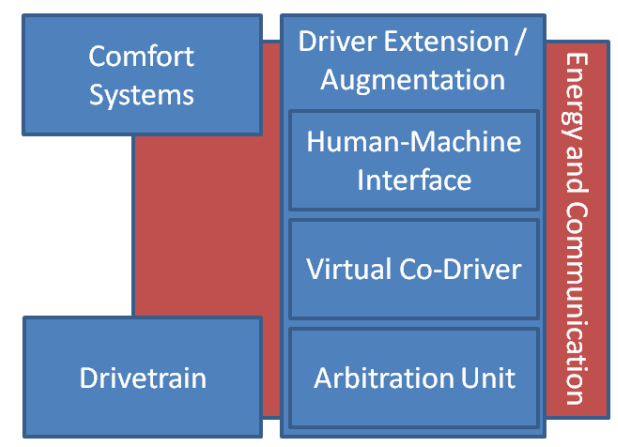

Figure 4. HMI Evolution inside the 5-Module approach

\section{SUGgested APPROACH}

The main advantage offered by the future-interfaces is extending the user into the environment, which takes place in two directions. The first one starts at the driver and is directed towards the environment: The personal mobility is brought to people who cannot operate a classical vehicle due to physical disabilities or the overall mental complexity of the task. By alleviating this issue, it becomes possible to manipulate the environment in new ways or on a finergrained scale. The other direction, from the vehicle and towards the driver, takes the multitude of sensors, which the intelligent vehicles are going to be equipped with, into account. The bulk of the data is fed to the assistance systems and other vehicle subsystems, but a selected amount is provided to the driver, in order to extend the user's own perception of the surrounding environment.

Fusion of interfaces and driver assistance is also happening in the same two directions. The interfaces relying on gazes, minimal hand and muscle movements or brain activity to name the few, continuously record data which is not related to primary vehicle operation task. This additional data, superimposed or acquired in parallel to the primary data used to transfer vehicle commands, provides a specific interface type with an inherent additional value for assistance systems. The user model therefore gets expanded with additional data sources, relevant primarily for the driver assistance. An example or data extraction for different interfaces is given in works [6], [4] and [5]. Since the human-machine interface is actively gaining data which is directly useful to the driver assistance, a fusion of two models is necessary in order to follow the principles of reducing the inter-module communication.

In order to reach the long-term vision of augmenting driver's capabilities, we placed emphasis on following topics:

\section{A. Horizontal Design with High Integration of HMI and DA}

We suggest a horizontal vehicle control paradigm, with an interpretation layer which can process context and react accordingly to the user interaction. This design principle additionally yields highly integrated human-machine interface components, like the virtual dashboard, constructed and tested in the project scope.

\section{B. Cross-Domain Transfer}

In order to ease the initial case studies and prototype construction, we make extensive use of technology transfer from adjacent domains. From the perspective of HMI for primary vehicle control, such are the medical and consumer electronics domains.

Sidestick steering systems have already been thoroughly investigated in the area of vehicles retrofitted for drivers with physical impairments. Such drivers cannot operate a typical automotive interface and require more integrated and less physically intensive input methods. Ergonomic studies and field tests in this area are transferred into our test vehicle.

Brain-Computer Interfaces were historically investigated in the medical domain. Recent developments however try to bring simplified devices into the consumer electronics field in order to operate applications with brain activity. It is important to draw a difference between interfaces which make heavy use of facial muscle activity and the ones which focus primarily on brain electric activity. For our purposes, it would be counter-intuitive to rely on muscle activity, so we focused on the methods which increase the accuracy of currently available devices.

\section{Suggesting the Transitional Steps}

Our goal is twofold: To increase the driving safety and to reduce the complexity of vehicle control. This goes in line with the previously mentioned reduction in kinetic energy necessary to operate the interface and increase in context processing on the vehicle side. An example of a braincomputer interface solution, a long-term vision which fully reduces the kinetic component of an interface, is given in the work of Birbaumer [8]. Building upon existing projections of technology development, we suggest a stepwise interface evolution model shown in table I. Each step introduces another level of processing complexity (assumption: 
processing power and signal fidelity increasing over time) and reduces the required kinetic energy. It is important that each transitional step provides additional value for the society, enabling sustainable focused research. For the driver input concepts, we have focused on the accessability and road safety. For the driver feedback concepts and control over various vehicle subsystems not related to primary vehicle steering, a touchscreen-based dashboard was selected. Such solution is widely recognized by the users due to the popularity of smartphone and tablets and has a large cross-domain potential from the domain of consumer electronics.

TABLE I. STEPWISE EVOLUTION OF INTERFACES FOR CONTINUOUS VEHICLE CONTROL

\begin{tabular}{|c|c|c|c|c|}
\hline & \multicolumn{4}{|c|}{ Evolution Steps } \\
\hline & $\begin{array}{l}\text { Current } \\
\text { Solution }\end{array}$ & $\begin{array}{c}\text { Immediate } \\
\text { Improvement }\end{array}$ & $\begin{array}{c}\text { Intermediate } \\
\text { Solutions }\end{array}$ & $\begin{array}{c}\text { Long-Term } \\
\text { Solution }\end{array}$ \\
\hline $\begin{array}{l}\text { Interface } \\
\text { Concept }\end{array}$ & $\begin{array}{l}\text { Steering } \\
\text { Wheel } \\
\text { and } \\
\text { Pedals }\end{array}$ & Sidestick & $\begin{array}{c}\text { Motor nerve } \\
\text { analysis }\end{array}$ & $\begin{array}{l}\text { Brain- } \\
\text { Computer } \\
\text { Interface }\end{array}$ \\
\hline $\begin{array}{l}\text { Added Value } \\
\text { for Driver } \\
\text { Assisstance }\end{array}$ & $\begin{array}{l}\text { Starting } \\
\text { point }\end{array}$ & $\begin{array}{c}\text { Force- } \\
\text { feedback for } \\
\text { both lateral } \\
\text { and } \\
\text { longitudinal } \\
\text { dynamics }\end{array}$ & Not analyzed & $\begin{array}{c}\text { Direct } \\
\text { analysis of } \\
\text { brainwave } \\
\text { activity }\end{array}$ \\
\hline $\begin{array}{l}\text { Added Value } \\
\text { for } \\
\text { Accessibility }\end{array}$ & $\begin{array}{c}\text { Starting } \\
\text { point }\end{array}$ & $\begin{array}{l}\text { Significantly } \\
\text { reduced } \\
\text { physical effort } \\
\text { to operate the } \\
\text { vehicle }\end{array}$ & $\begin{array}{c}\text { Driving } \\
\text { possible } \\
\text { without } \\
\text { actual body } \\
\text { movements }\end{array}$ & $\begin{array}{c}\text { Driving } \\
\text { possible } \\
\text { without } \\
\text { any motor } \\
\text { nerve } \\
\text { activity }\end{array}$ \\
\hline
\end{tabular}

\section{Suitable Software Platforms and Standards for the First Transitional Step}

Reducing and standardizing the basic data transferred to and from the HMI and DA was the first step in overall system design. In the second, we go one level and try to define a standard set of software platforms and technologies used for the first transitional step. Easy integration with internet-of-things and load balancing (parameterizing the amount of data processing done on HMI aggregates) were regarded as a bonus in the selection process. We settled on Nokia/Digia QT and HTML-based solutions, since they fulfilled all the requirements. For our sensor data management model, we leaned on the Open Geospatial Consortium (OGC) Sensor Web Enablement (SWE) standards [7].

\section{E. Choosing Models for the Context Description}

In the scope of the HMI development, we have focused on two levels of system modeling. The first one includes modeling data-flow without safety-relevant requirements and is already described as being solved by SWE. The other level focuses on retaining a constant snapshot of the all relevant variables describing the system state. For this model, we have opted for a probability network variant of the junction tree. This model contains all the data relevant for driver assistance decisions. On-line addition and removal of nodes is quite easy and without almost any performance penalty. Complexity of inference on a set of well-defined questions relevant for driver assistance is low by design, since it relies on junction trees for exact inference [9].

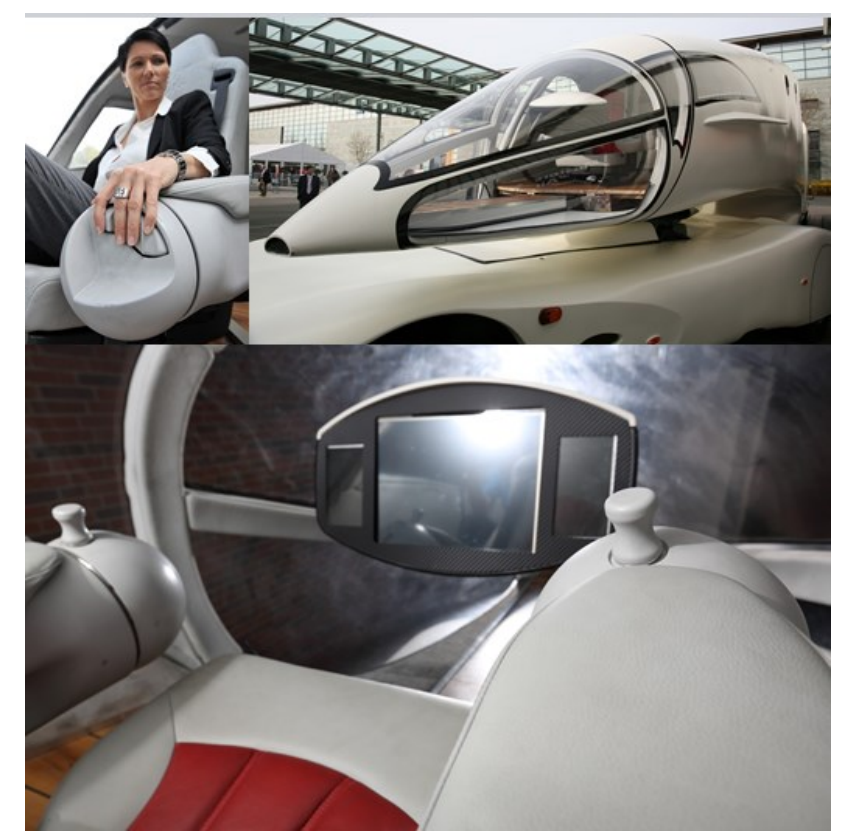

Figure 5. Various views into the cockpit and sidestick control. Fotos by Derek Schuh / Schwäbische Zeitung und Medienfabrik Gütersloh.

\section{PRototype VehicLE}

As previously explained, our prototype vehicle lays down the basics of the horizontal approach in interface design. It features a transitional interface with a touchscreen console and two sidesticks. Figure 5 shows the entire driver's workplace in greater detail. A custom-made driver's seat with integrated sidestick controls (two modules) has been installed. The units support force feedback and are paired for redundancy. Lateral vehicle control (steering) is kept physically separated from the longitudinal (acceleration and braking), but both are controlled with one hand. A parking and maneuver assistant has been developed to reduce training time for the sidesticks and increase overall safety. The application is shown in figure 6.

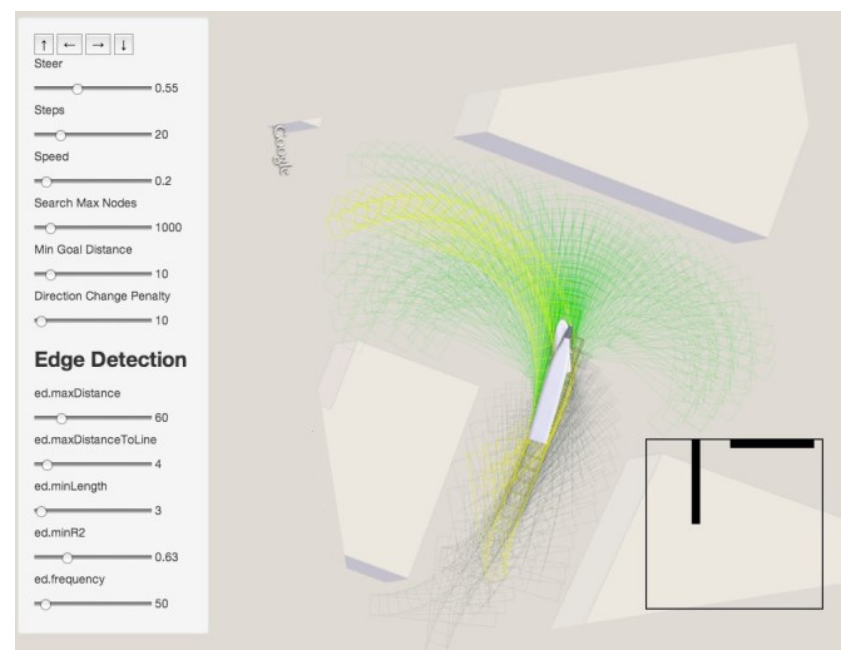

Figure 6. Parking assistance application in the configuration mode. 
We have constructed a central console element and implemented a QT-based virtual dashboard, augmented with the webkit-based parking and maneuver assistance and a webkit-based frontend for in-vehicle energy and data flow visualization. The dashboard is shown in the figure 7.

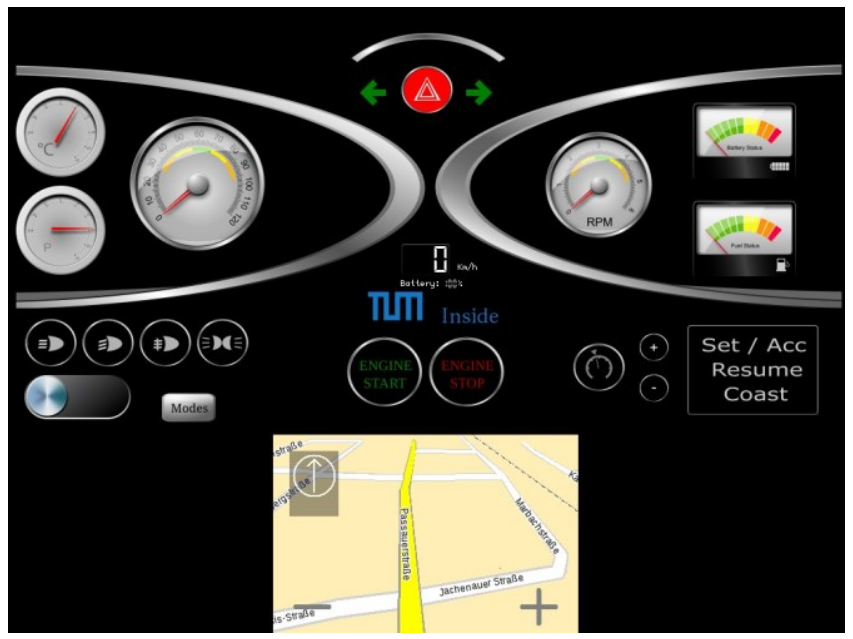

Figure 7. One of the interface "skins" on the central console.

It features all the classical indicators as well as live camera feed from the vehicle left and right sides and the navigational software. Basic control of vehicle lighting and engine was implemented through a CAN bus connection to the external embedded processing unit. Low-level integration with other software applications and hardware drivers was successfully tested, one example being the Navit navigational software. Personalized interfaces for different users based on XML profiles were implemented and tested. In short, the method of choice provided us with intuitive interface design tools and full portability, but low-level access to underlying hardware was also possible. This proved essential for prototype development and concept testing, as compared to other, more popular but closed platforms.

Both applications for the dashboard are running on the same software platform, the previously mentioned webkit. They represent a mixture of human-machine interface and driver assistance applications, which can embedded into a vehicle or scaled down onto a personal mobile device.

The frontend for data visualization provides basic data about the vehicle status on the screens inside the vehicle and on connected smartphones and tablets. It is an informative application, requiring little user manipulation. A screenshot is given in figure 8 .

The parking and maneuvering assistant shifts the emphasis in favor of manipulation again, but adds additional level of complexity inherent to path planning and collision detection. This part of the application can be offloaded to more powerful processing units inside the vehicle.

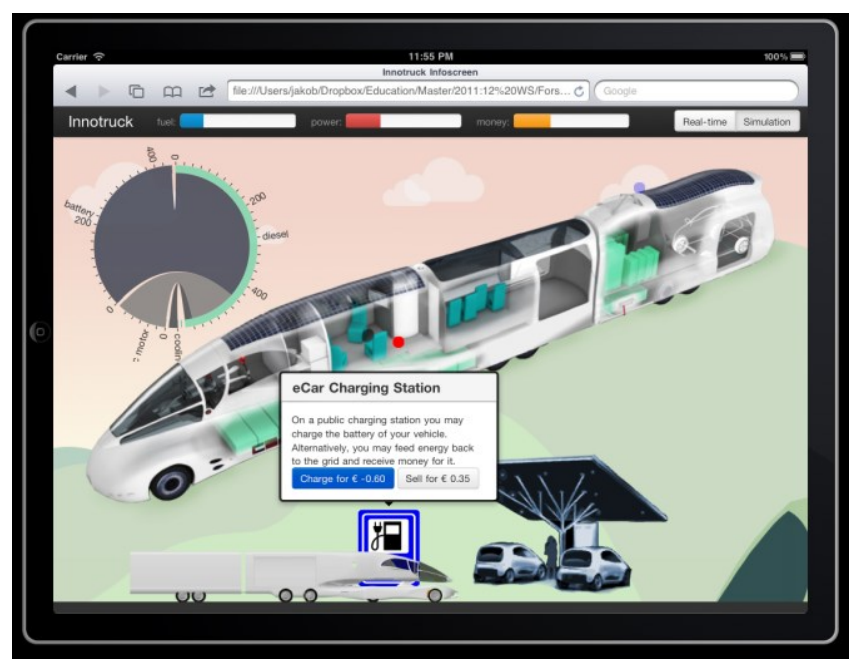

Figure 8. Data and energy flow visualization application.

\section{DRIVING SIMULATOR}

In order to perform data acquisition for driver assistance on a larger scale, we modified an existing Vires Virtual Test Drive simulator, complete with a vehicle mock-up. The simulator has been retrofitted with a simple joystick and EEG-based brain-computer interface. It is shown in figure 9 during one of the test runs. Sidestick and EEG data has been collected and analyzed during test drivers with experimental subjects. The goal was to make assumptions on their mental and physical state, but also to make blind guesses on the type of simulated environment (driving inside or outside the city). The EEG is a unit from the consumer electronics domain, designed with an emphasis on ergonomics and ease of handling. Operating the vehicle with the BCI was not the primary concern, since it has already been demonstrated by the AutoNOMOS Labs [10].

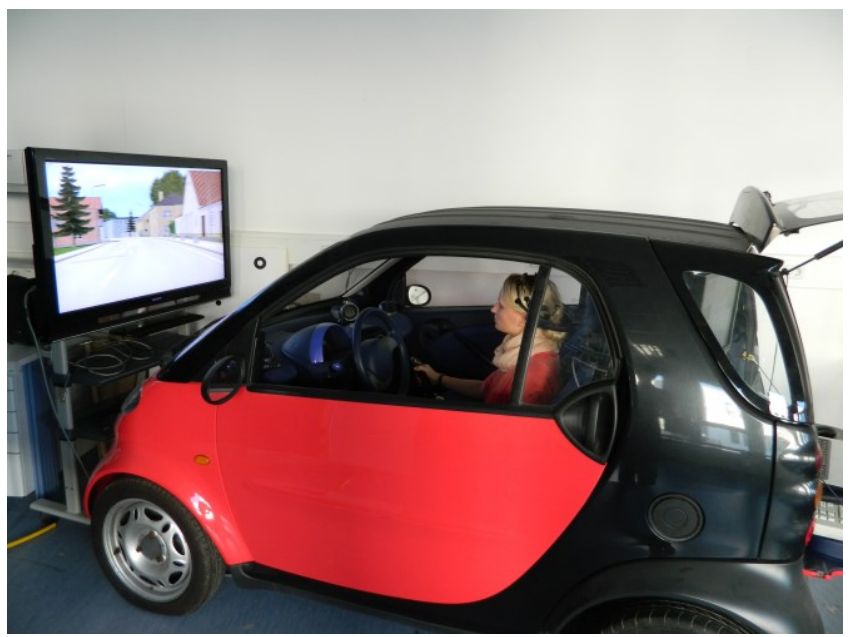

Figure 9. The driving simulator mock-up built upon VIRES Virtual Test Drive.

We concentrated on passive data collection and determining of user's fatigue. We have shown that such analysis is possible with the current state of consumer electronics devices and that the device does not obstruct the 
driver in any significant way [4].

\section{CONCLUSION}

The transition to future interfaces requiring low physical involvement and providing high informational content offers additional value for both driver assistance and accessibility. Factors like population aging, urban growth and vehicle personalization can be leveraged to further ease market entry for new technologies. The biggest benefit we see from the anticipated change is the augmentation of the human abilities through an intelligent vehicle which processes entire interaction context. The vehicle concept of the future can be refined into a cooperative robotic agent, assisting the user safely located inside the system and erasing the border between automobiles and robotic agents. We suggest the following steps in order to facilitate and properly manage this shift:

- Horizontal design with an interpretation layer with context processing and highly integrated HMI aggregates

- Using cross-domain transfer to accelerate the prototype design, acceptance and data acquisition

- Specifying transitional steps to the long-term vision which have a direct added value for the society

- Specifying software platforms and standards for the first transitional step, enabling rapid implementation

- Choosing and expanding context models which capture all important factors of a two future fused systems-ofsystems: the user, vehicle and the environment on one side and HMI and DAS on the other

\section{ACKNOWLEDGMENT}

We would like to thank the International Graduate School of Science and Engineering (IGSSE) as well the Institute of Advanced Study (IAS) of the Technische Universität München for all the support in the organization of our project. Furthermore, I would like to thank fortiss, an Institut der Technischen Universität München, for all the constructive advice and practical support.

\section{REFERENCES}

[1] Innotruck Test Drive, 2012, Daily Planet Episode November 3rd-5th Part 2, Karlsdorf-Neuthard, Discovery Channel

[2] A. Amditis, L. Andreone, A. Polychronopoulos, J. Engström, "Design and Development of an Adaptive Integrated Driver-Vehicle Interface: Overview of The AIDE Project" Proc., IFAC 16th World Congress, Prague, Czech Republic, 4 - 8 July 2005

[3] Buckl, C.; Camek, A.; Kainz, G.; Simon, C.; Mercep, L.; Stahle, H.; Knoll, A.; , "The software car: Building ICT architectures for future electric vehicles," Electric Vehicle Conference (IEVC), 2012 IEEE International, vol., no., pp.1-8, 4-8 March 2012

[4] Ljubo Mercep, Gernot Spiegelberg, Alois Knoll, A robust driver assessment method for the brain-computer interface, IADIS Interfaces and Human Computer Interaction 2013, Prague. In Review.

[5] Lei Cao; Jie Li; Yaoru Sun; Huaping Zhu; Chungang Yan; , "EEGbased vigilance analysis by using fisher score and PCA algorithm," Progress in Informatics and Computing (PIC), 2010 IEEE International Conference on, vol.1, no., pp.175-179, 10-12 Dec. 2010
[6] Ljubo Mercep, Gernot Spiegelberg, and Alois Knoll. „A gameoriented approach to driver state recognition from sidestick input". In 6. VDI/VDE Fachtagung USEWARE 2012 - Mensch-MaschineInteraktion, pages 3-8, Deutsches Forschungszentrum für Künstliche Intelligenz DFKI Kaiserslautern, December 2012.

[7] Jakob Stoeck, Ljubo Mercep, Gernot Spiegelberg, and Alois Knoll. Platform-independent interface for the management of sensorgenerated power and data flows in an automotive data-centric architecture. In 6. VDI/VDE Fachtagung USEWARE 2012 - MenschMaschine-Interaktion, pages 33-36, Deutsches Forschungszentrum für Künstliche Intelligenz DFKI Kaiserslautern, December 2012.

[8] J. R. Wolpaw, N. Birbaumer, D. J. McFarland, G. Pfurtscheller, T. M. Vaughan, "Brain-Computer interfaces for communication and control," in Clinical Neurophysiology vol. 113, 2002, pp. 767-791.

[9] H. Pan, D. McMichael, M. Lendjel, "Information Fusion, Causal Probabilistic Network And Probanet II: Inference Algorithms and Probanet System", Proc. 1st Intl. Workshop on Image Analysis and Information Fusion, pages 445-458, 1997

[10] Brain Driver 2012, AutoNOMOS labs Videos, Berlin, online video, accessed 15 November 2012, < http://www.autonomos.inf.fuberlin.de/media/videos>. 